\section{Section News}

Interdisciplinary Approaches to International History and Politics

Interdisciplinary Approaches to International History and Politics is pleased to announce the launch of its web site (www.asu.edu/clas/ polisci/ihap/), which features syllabi and meeting information. Also, information on joining IHAP was omitted from the APSA membership form in the March 2000 issue of $P S$. APSA has apologized for this oversight. Section dues are $\$ 5$ a year and interested individuals are encouraged to use the form in this issue of the journal to request section membership.

\section{Political Methodology}

Annual dues for membership in Political Methodology, which include subscription to Political Analysis, are $\$ 25$. This amount was incorrectly listed on the membership form included in the March 2000 issue of $P S$.

\section{Foundation of Political Theory}

The Foundations home page can now be found at www. political-theory.org. The site features section-related material, including copies of current and previous newsletters, and links to theory-oriented sites. Visitors can also view and purchase recently published works in political theory through a link with Amazon.com.

\title{
Recent Contributors to APSA Awards and Programs
}

Paul Allen Beck

Henry Bienen

Gerard Patrick Bushell

Thomas M. Callaghy

Clarke E. Cochran

Larry Diamond

Alexander A. Doska

Delmer D. Dunn

Cynthia H. Enloe

Victoria A. Farrar-Myers

Christopher H. Foreman, Jr.

William E. Frenzel

Jose E. Garriga-Pico
Clark C. Gibson

Peter T. Higgins

Juan Carlos Huerta

Burdett A. Loomis

Walter G. Markham

Frank O. Mora

James S. Pacy

Wilbur C. Rich

Molly Lyndon Shanley

Joseph M. Schwartz

Marc A. Triebwasser

David M. Wilson

Laura R. Woliver

Kazuo Yamauchi

\section{Thank You!}




\section{the centennial campaign}

\section{celebrating the past by investing in the future}

\author{
Centennial Circle \\ $(\$ 25,000+)$ \\ Walter E. Beach - C, A, B, Be, M \\ Doris Graber \\ Pendleton Herring \\ Huang Hsing Foundation \& Chun-tu \\ Hsueh - $\mathrm{H}$ \\ University of Louisville, in honor of \\ Aaron Wildavsky \\ Elinor \& Vincent Ostrom, in honor of \\ Alma Ostrom \& Leah Hopkins Awan \\ $-O$ \\ Estate of Leo A. Shifrin \\ Frank J. Sorauf - C, M \\ 1903 Circle \\ $(\$ 15,000+)$ \\ Richard F. Fenno, Jr. - PF \\ Jo Freeman - W \\ Arend Lijphart \\ Norman H. Nie \\ Jack W. Peltason - C, A \\ 2003 Circle \\ $(\$ 10,000)$
}

Ruth S. Jones \& Warren E. Miller -

C, M, A

Nannerl \& Robert O. Keohane - Br, $B, L$

Dale Rogers Marshall

Pi Sigma Alpha

Founders Circle

$(\$ 5,000+)$

Anthony Affigne - B, L

Lucius J. Barker

Robert H. Bates

Susan C. Bourque

David Brady

Roger H.\& Nancy Davidson - C, A, S

Luis Ricardo Fraga - C, L

James L. Gibson

Betty Glad - P

John Mark Hansen

Martin O. Heisler - A

Jennifer P. Hochschild \& C. Anthony

\author{
Broh - C, B \\ Matthew Holden, Jr. \\ Gary C. Jacobson \\ Malcolm E. Jewell \& Sarah M. \\ Morehouse - C, M \\ Joyce K. Kallgren \\ Mary Fainsod Katzenstein \& Peter \\ Katzenstein \\ Martha Joynt Kumar, in honor of \\ Ann Devroy - $P$ \\ Ruth P. Morgan - P \\ Norman J. Ornstein \\ Jewel L. Prestage - PF, B \\ Lucian W. Pye \\ J. Austin \& Nancy Ranney \\ Catherine $E$. Rudder - $C, A, M, B$, \\ $B r, W, L$ \\ J. Merrill Shanks - M \\ William O. Slayman - Br, M \\ Paul M. Sniderman \\ James $Q$. Wilson \\ Thomas R. Wolanin - S \\ Nancy $\mathrm{H}$. Zingale \\ Builders Circle \\ $(\$ 2,500+)$ \\ David Adamany \\ James $E$. Anderson \\ Anonymous - $B$ \\ Patricia Artinian - A \\ William I. Bacchus \\ Michael A. Baer \\ Barbara B. Bardes \\ Paul Allen Beck - C, A, M \\ John F. Bibby \\ John G. Bretting, in honor of F. Chris \\ Garcia - L \\ Charles S. Bullock, III \\ Philip E. Converse - C, M \\ William J. Daniels \\ Christopher J. Deering - C, S \\ Jorge I. Dominguez \\ Lawrence C. Dodd, in honor of the \\ Department of Political Science, \\ University of Minnesota \\ Robert F. Durant - V \\ Leon D. Epstein - C, M
}

as of May 1, 2000

Heinz Eulau - C, A

Kathleen A. Frankovic

Virginia H Gray - C, A

Joel B. Grossman

Charles D. Hadley - C, A

Robert J-P. Hauck - C, A, M

Anne Hopkins

Robert Huckfeldt

$M$. Kent Jennings

Robert Jervis

Loch K. Johnson

Charles O. Jones - C, A, P

William J. Keefe

Rita Mae Kelly - B

Herbert P. Kitschelt

Allan Kornberg

Kay Lawson

Avery Leiserson

Burdett A. Loomis

Susan A. MacManus - C, A, B, V

David B. Magleby - $S$

Thomas E. Mann

David R. Mares - L

Joel Margolis - $\mathrm{S}$

Valerie J. Martinez-Ebers - L

David R. Mayhew

Daniel A. Mazmanian

Paula D. McClain

Cynthia McClintock

Lorraine M. McDonnell \& M.

Stephen Weatherford - P

Jeffrey R. Miller \& Margot L. VanDis Karen O'Connor

David Scott Palmer, in memory of

Eldon "Bud" Kenworthy

Leonard Parkinson

James J. Prestage - PF

Michael B. Preston - C, PF

George H. Quester

Beryl A. Radin

Randall Ripley

Alan Rosenthal

Kay Lehman Schlozman - C, B

Mildred A. Schwartz - C, M

Lee Sigelman

Howard J. Silver

Barbara Sinclair

Names in italics are new gifts since the last publication of the Giving Circles list.

$\mathrm{C}=$ General Campaign Fund, $\mathrm{A}=$ Artinian Fund, $\mathrm{B}=$ Barnett Fund, Be = Beach Fund, $\mathrm{Br}=\mathrm{Bryce}$ Fund, $\mathrm{H}=\mathrm{Hsueh}$ Fund, $\mathrm{L}=$ Latino Fund, $M=$ Miller Fund, $\mathrm{O}=$ Ostrom Fund, $\mathrm{P}=$ Presidency Research Fund, PF = Prestage-Fenno Fund, $\mathrm{V}=\mathrm{Volcker}, \mathrm{W}=$ Women \& Politics Fund 


\section{the centennial campaign celebrating the past by investing in the future}

Raphael J. Sonenshein Harvey Starr

Dorothy McBride Stetson Joseph Stewart, Jr. - C, PF Judith Hicks Stiehm - C, A

James A. Stimson - M

Camilla M. Stivers - V

Clarence N. Stone

Edward Thompson, III, in honor of Marguerite Ross Barnett \& Vincent J. Browne - B

James $A$. Thurber

David B. Truman Sidney Verba

John C. Wahlke - C, A, P

Charls E. Walker

Kenneth N. Waltz

Paul J. Weber.

Susan Welch

David Wilsford, in honor of Warren Miller - C, M

Lois R. Wise - V

\section{Second Century Society}

$(\$ 1,000+)$

Herbert Alexander - A, M

Hayward R. Alker

Gabriel Almond

Herbert B. Asher

Manual Avalos, in memory of

Latticia Galindo - L

Samuel $H$. Barnes - A, M

Samuel $H$. Beer

Jeffrey M. Berry

James W. Bjorkman

Christopher J. Bosso, in honor of the

Political Science faculty at the

University of Pittsburgh

Richard W. Boyd \& Martha Crenshaw

Steven J. Brams

Michael A. Brintnall

Nancy Brune, in memory of Roger R. Brune - L

Frances Burke

James MacGregor Burns

Lief $\mathrm{H}$. Carter
Leonard Champney - A

Eugene Y.S. Chang - S, $P$

Daniel S. Cheever, in memory \& in

honor of Ralph Bunche - PF

The Claremont Institute - Be

Clarke E. Cochran

Thomas E. Cronin - C, P

Milton C. Cummings, Jr.

Robert A. Dahl

Louis DeSipio - L

Jane E. Fountain, in honor of Robert

C. Wood - V

Linda Fowler

H. George Frederickson - V

Jurg Martin Gabriel

F. Chris Garcia - L

Louis C. Gawthrop - A

George J. Graham, Jr. - A

Susan W. Hammond

Roderick P. Hart - C, P

C. Randall Henning

Mary A. Hepburn, in honor of Sheilah Mann

Rodney E. Hero - L

Robert J. Huckshorn

William Hudson - A

Robert P. \& Dixie S. Huefner, in

honor of Edward Banfield

Karen M. Hult - $P$

Dennis Ippolito

Harold \& Jean Jacobson - M

Bruce W. Jentleson

Mark P. Jones - M

Michael A. Jones-Correa - $L$

Nolan E. Jones, in honor of Adolph

Reed, Sr. - B, PF

David C. Leege - M

Margaret Levi \& Robert Kaplan

Gerhard Loewenberg

Benjamin Marquez - $L$

Janet $M$. Martin - $P$

William Mishler, in honor of Allan

Kornberg - C, A, S

Robert Montjoy, in honor of York Willbern - C, $V$

Lisa J. Montoya - L

Lois B. Moreland - PF as of May 1, 2000

John S. Odell

Kenneth T. Palmer

Desiree Pedescleaux - A, PF

Nelson W. Polsby - A

Henry Pratt, in honor of David Truman

David E. Price

Lyn Ragsdale, in memory of Barbara Hinckley - $P$

Thomas F. Remington

Leroy N. Rieselbach - S

Ronald J. Schmidt, Sr. - L

Gary M. Segura - L

Sally Selden - V

Donna E. Shalala - B

W. Phillips Shively - M

Christine Marie Sierra - L

Roberta S. Sigel - C, B

Elliot E. Slotnick, in honor of Frank J. Sorauf

Thomas A. Spragens, Jr.

Janet D. Steiger - S

Sybil L. Stokes

Gerald H. Stollman

Deborah Stone - C, B

Raymond Tatalovich - $P$

Arturo Vega, in honor of the Political

Science Department at St. Mary's

University, San Antonio, TX, and the

Carl Albert Congressional Research

Center, University of Oklahoma - L

Charles $E$. Walcott - $P$

Stephen L. Wasby - A, B, M, S - in

memory of Morris Udall

Herbert F. Weisberg

University of Wisonsin-Milwaukee

Political Science Department, family

$\&$ friends, in honor of Cornelius $P$. Cotter

Raymond $\mathrm{F}$. Wolfinger, in honor of Warren E. Miller - M

Maurice C. Woodard - C, B, PF James $V$. Young

Robert L. Youngblood - $M$

Joseph F. Zimmerman

Names in italics are new gifts since the last publication of the Giving Circles list.

$\mathrm{C}=$ General Campaign Fund, $\mathrm{A}=$ Artinian Fund, $\mathrm{B}=$ Barnett Fund, $\mathrm{Be}=$ Beach Fund, $\mathrm{Br}=\mathrm{Bryce}$ Fund, $\mathrm{H}=\mathrm{Hsueh}$ Fund, $\mathrm{L}=$ Latino Fund, $M=$ Miller Fund, $O=$ Ostrom Fund, $P=$ Presidency Research Fund, $P F=$ Prestage-Fenno Fund, $V=V o l c k e r, ~ W=$ Women \& Politics Fund 


\section{the centennial campaign celebrating the past by investing in the future}

Anniversary Society

$(\$ 500+)$

Joel D. Aberbach, in honor of Robert Lane

Alan I. Abramowitz

Martha Ackelsberg - B

American Review of Public

Administration - $\mathrm{V}$

Association of Chinese Political Studies

Judith A. Baer - B

Frank R. Baumgartner \& Gretchen G.

Casper, in memory of Jack $L$.

Walker, Jr.

Frances Stokes Berry - V

Henry Bienen

Janet K. Boles - B, P

Ann O'Meara Bowman

Richard A. Brody - M

Emmett Buell - A

Bruce Bueno de Mequita

David A. Caputo

David Collier \& Ruth Berins Collier

M. Margaret Conway Joseph Cooper

Gary W. Cox

C. Jeremy Curtoys

Vincent \& Anne Davis, in honor of

Charles 0 . Jones

Linda \& Peter deLeon - $V$

George C. Edwards, III - P

Lisa Garcia Bedolla - L

John B. Gates, in memory of Richard Sinopoli

Harvey Glickman, in memory of $\mathrm{H}$. Hubert Wilson

Kenneth \& Amanda Goldstein - M

Robert T. Golembiewski

Robert C. Grady - A

Melinda Gann Hall - A, B

Carol Hardy-Fanta

William A. Hazleton \& Sandra WoyHazleton

John R. Hibbing

Kim Quaile Hill - M

John W. Holcombe, in memory of George S. Blair
Patricia A. Hurley - M

Patricia W. Ingraham - V

W. Landis Jones

Peter Juviler - A

Miles Kahler

University of Kansas, Dept of Public

Administration faculty colleagues of

$H$. George Frederickson - V

Donald F. Kettl - A, V

Samuel A. Kirkpatrick - M

Gregory B. Lewis - V

Mark Lichbach

Serge E. Logan

David L. Lowery

Naomi B. Lynn - B

L. Sandy Maisel, in honor of Warren

E. Miller - M

Lynn Mather - B

Nancy E. McGlen - C, A, W

$H$. Brinton Milward, in memory of

Charles $\mathrm{H}$. Levine $-\mathrm{V}$

J. Donald Moon

Robert J. Mundt

Richard W. Murray

Richard E. Neustadt - $P$

Pippa Norris - M

Joseph S. Nye, Jr.

Rosemary O'Leary - $V$

Dorothy A. Palmer, in memory of

Raymond L. Lee - A

Carole Pateman - A

Mark P. Petracca, in honor of Gina \& Joseph Petracca

Susan J. Pharr

Gerald M. Pomper - A

Robert D. Putnam

Donald L. Robinson

Bert A. Rockman - A, V

John A. Rohr - V

Lloyd I. \& Susanne Hoeber Rudolph - C, B

Virginia Sapiro

Joanna Vecchiarelli Scott - W

Molly Lyndon Shanley - B

Robert Y. Shapiro - P

Beth Simmons as of May 1, 2000

Harold Stanley - A

Robert T. Starks, in honor of Jewel

Prestage - $P F$

Randall Strahan

Norman C. Thomas - $\mathrm{P}$

Susan Tolleson-Rinehart Jeffrey $K$. Tulis - $P$

Frederick M. Wirt - B

Kenneth K. Wong

Diel S. Wright

David Vogel

\section{Campaign Friends}

$(\$ 250+)$

J. Theodore Anagnoson

Kristi Andersen - C, B

Donald G. Balmer

Harold F. Bass, Jr., in memory of

Cornelius Cotter - C, A

John C. Berg - A

Gayle Binion

Jeanne C. Blamey

Mary Anne Borrelli - P

Randall L. Calvert

David T. Canon - S

John A. Clark - C

Conference Group on Taiwan Studies $-\mathrm{Br}$

Constance Ewing Cook

Cary R. Covington - $P$

Russell Dalton - A

John G. Geer - M

Joyce Gelb - C, B

Michael Genovese - P

Marissa Martino Golden - $V$

Fred I. Greenstein - C, P John W. Harbeson

Robert A. Heineman - A

Marjorie R. Hershey - A Allen D. Hertzke

Raymond F. Hopkins

$E$. Terrence Jones - $M$ Marion R. Just - A

Nancy Kassop - PJanuary 3, 2000

William R. Keech - A Scott Keeter - A

Names in italics are new gifts since the last publication of the Giving Circles list.

$\mathrm{C}=$ General Campaign Fund, $\mathrm{A}=$ Artinian Fund, $\mathrm{B}=$ Barnett Fund, $\mathrm{Be}=$ Beach Fund, $\mathrm{Br}=\mathrm{Bryce}$ Fund, $\mathrm{H}=\mathrm{Hsueh}$ Fund, $\mathrm{L}=$ Latino Fund, $M=$ Miller Fund, $O=$ Ostrom Fund, $P=$ Presidency Research Fund, $P F=$ Prestage-Fenno Fund, $V=V o l c k e r, ~ W=$ Women \& Politics Fund 


\section{the centennial campaign}

\section{celebrating the past by investing in the future}

\author{
Frank Kessler - $\mathrm{P}$ \\ John Kincaid \\ Samuel Krislov \& Judith Gillespie - A \\ David A. Lake \\ David D. Laitin \\ Logan A. Lee \\ Jacob T. Levy \\ Linda Lopez - L \\ Andrew S. McFarland - A \\ Roger A. McGuire - S \\ Eugene Meehan - A \\ Cheryl M. Miller - PF \\ Bruce Miroff - A \\ New England Political Science \\ Association - A \\ Richard G. Niemi \\ Susan S. Northcutt \\ James L. O'Sullivan - Br \\ Don Racheter \\ David M. Rayside \\ Richard \& Rosemary Rose - A \\ Jo Ellen Ross - PF \\ Herbert J. Rubin - A \\ Arlene W. Saxonhouse - M \\ W. Rand Smith \\ Carol M. Swain - C, A, B \\ Santa \& Michael Traugott - $M$ \\ Leo A.W. Wiegman - A \\ Clyde Wilcox \\ Shirley Anne Warshaw - $P$ \\ Robert C. Wood - A, P \\ M. Crawford Young \\ Campaign Associates \\ (\$100-\$249) \\ Henry J. Abraham \\ Paul. R. Abramson - M \\ Shariff Ahmad - S \\ Gar Alperovitz \\ Asher Arian - A \\ David J. Bellshaw - M \\ David R. Berman \\ Nancy Bermeo - B \\ Janet Box-Steffensmeier - C, A, B, \\ $M, S$ \\ Evelyn S. Brewster - M
}

Amy Bridges - $L$

Thomas M. Callaghy

Colin Campbell - A

Ellen Carnaghan

Susan J. Carroll - B

$N$. Joseph Cayer - V

Tyson Chaney - C, V

Celia F. Cohen - S

Jeffrey E. Cohen - $P$

William J. Crotty

I.M. Destler - A

Donna Robinson Divine

Michael W. Doyle \& Amy Gutmann

Malcolm Feeley - A

Paul Ferber

James W. Fesler - A, V

Jane Flax - B

John P. \& Lorraine W. Frank - M

Esther Fuchs - B

Emily R. Gill - B

Girls Ranch, Inc. of Arizona - M

Nicole A. Gordon - M

Samuel K. Gove - V

Joanne Gowa

Joseph M. Grieco

Kenneth A. Heath - S

Charles P. Henry - B, PF

Richard and Cheryl Herrera - M

Paul S. Herrnson - A

Harry N. Hirsch

Kenneth Hoover, in memory of

Edward Artinian - A

R. Gordon Hoxie - $P$

Serge Hurtig - C, M

James Jennings - $L$

Jim Moss \& Company - M

John R. Johannes - $S$

Tobe Johnson - PF

Mary Donovan Jones - $M$

Melvin A. Kahn - M

Ronald Kahn

Ellis Katz

Lael R. Keiser - V

Christopher Kelaher - A

James L. Kingsland - PF

John J. Kirlin as of May 1, 2000

Michael G. Krukones

Susan S. Lederman - A

Michael H. Levin - S

Sarah F. Liebschutz, in memory of

Peter Aranson \& Daniel Elazar Michael Lienesch

Roderick MacFarquhar

Harvey C. Mansfield, Jr. - A

Scott L. McLean - A

Wilson Carey McWilliams - A

Gabriel Miller - A

Harris N. Miller - S

Kristen Monroe

David R. Morgan - A, V

Charles C. Moskos, Jr.

Elizabeth F. Moulds

Susanne D. Mueller - B

Eric J. Narcisse - C, PF

Carol Nechemias

David Newman - A

Lela G. Noble - B

Joseph L. Nogee

Helmut Norpoth - A, M Diana M. Owen

Marian Lief Palley - B

Robert A. Pastor

Samuel C. Patterson - A

Thomas E. Patterson - A

William D. Pederson Jack Pfister - $M$

John C. Pierce - M

Dianne Pinderhughes - $B$ Richard Pious - $P$

Portal Pine Creek Canyon

Homeowners Assocation Unit III - M Jorgen Rasmussen - A

Representation \& Electoral Systems Organized Section

Margaret A. Robinson - M

Nancy L. Rosenblum

Frances M. Rosenbluth

Margaret Ann Rosenthal - A Marc Howard Ross Mark Sawyer - PF

Anne L. Schneider - B Harold Seidman - V 


\title{
the centennial campaign
}

\section{celebrating the past by investing in the future}

as of May 1, 2000

\author{
Byron E. Shafer - A \\ Kenneth A. Shepsle - M \\ Steven A. Shull - P \\ Sheldon W. Simon - M \\ Carolyn M. Shrewsbury - B \\ Peter N. Skerry \\ Theda Skocpol - B \\ Etel Solingen \\ Albert Somit \\ Robert J. Spitzer - A \\ Phillippa Strum - B \\ Kathleen Tapport - B \\ Leslie Paul Thiele - A \\ Craig Thomas $-V$ \\ Emma S. Thomas - $M$ \\ Joan Hulse Thompson - B \\ J. Ann Tickner - B \\ R. Lawrence Vandenberg \\ Kenneth D. Wald - A \\ Herbert Waltzer - A \\ Robert E. Ward \\ Marcia Lynn Whicker, in honor of \\ Malcolm Jewell - A \\ Stephen White - A \\ Donald \& Margaret Wickler - M \\ Clyde Wilcox - A \\ Kay Williams - M \\ Patrick J. Wolf - V \\ Stephen B. Wood \\ Aristide R. Zolberg - $B$ \\ Alan Zuckerman - A \\ Contributors \\ (\$0-\$99) \\ Karen J. Alter \\ Gloria C. Anglon - L \\ Paul E. Arnold \\ David M. Barrett - P \\ Donald C. Baumer \\ Cristina Beltran - L \\ Seyla Benhabib \\ Frederic A. Bergerson \\ Thomas E. Borcherding \\ Evelyn Z. Brokin \\ John E. Brown - M \\ Frances Burke - A \\ Keith Bybee - PF
}

\author{
Sara G. Carpenter - M \\ Brenda W. Carter - A \\ Allan J. Cigler - A \\ Caleb M. Clark - A \\ Patrick Coby \\ William F. Connolley, Jr. \\ Michael J. Coppedge \\ Alex N. Dragnich - A \\ Pat M. Dunham - A \\ Valerie Earle - $M$ \\ Robert $M$. Eisinger - $P$ \\ Cynthia Enloe - A \\ Timothy Fuller \\ Peggy Glick - M \\ Naomi \& David Goodell \\ Lawrence Graham - A \\ Martin Gruberg - B \\ Glen Halva-Neubauer \\ Roger Hamburg - A \\ Jonathan Hartlyn \\ Barbara J. Hayler - B \\ Alice L. Hearst \\ Charles F. Hermann \\ Frederick M. Herrmann - M \\ Dean R. Hewitt \\ Erik P. Hoffmann - A \\ Susan E. Howell \\ Malcolm E. Jewell - A \\ Charlotte Joseph \\ Junko Kato \\ Herbert Kaufman - A \\ Richard C. Kearney - A \\ Anthony King - A \\ Henry Krisch - A \\ Lan Thuc Le \\ Jerome S. Legge, Jr. - A \\ Lance Leloup \\ Jonathan Lemco - A \\ Elaine S. Levine - A \\ Jeremy R.T. Lewis - A \\ Carl Lieberman - $P$ \\ Robert C. Lieberman - A \\ Pei-te Lien - L \\ Charles H. Lipson \\ Lyceum Books, Inc. - A \\ Elaine P. Maimon - M \\ Michael Margolis - A
}

\author{
Suzanne M. Marilley - C, B \\ Mark G. Mazzie - M \\ Margaret \& Ken McBeth - M \\ Clyde D. Mckee, Jr. - A \\ Richard L. Merritt - A \\ Stuart S. Nagel - A
}

Univ of North Carolina, Chapel Hill,

Dept. of Political Science - M

Anne M. Parsons

Mark A. Peterson - A

Edward B. Portis - A

Wendy M. Rahn - A

Ronald B. Rapoport - S

Paul Rejai - A

Ross R. Rice - M

Wilbur C. Rich - B

David B. Robertson - B

Bernard H. Ross - A

Blair Ruble

Wilma Rule - B

Cynthia \& Donald Ryan

Laura J. Scalia - A

Beth S. Schapiro

Jean Reith Schroedel - B

L. Earl Shaw, Jr. - A

Susan J. Shearon - M

Masaki Shigeta

Henry B. Sirgo - A

Theda Skocpol - B

Rosemary Glass Spalding - M

Robert J. Spitzer - A

Alonzo T. Stephens - PF

Kathryn Dunn Tenpas

Robert \& Martha Thompson - M Charles B. Turpin

Henry Valen - M

Diane E. Wall - B

Retha W. Warnicke - M

Marvin G. Weinbaum - A

Gregory W. White

Janet L. Womack

Iris Marion Young

Names in italics are new gifts since the last publication of the Giving Circles list.

$\mathrm{C}=$ General Campaign Fund, $\mathrm{A}=$ Artinian Fund, $\mathrm{B}=$ Barnett Fund, $\mathrm{Be}=$ Beach Fund, $\mathrm{Br}=$ Bryce Fund, $\mathrm{H}=$ Hsueh Fund, $\mathrm{L}=$ Latino Fund, $M=$ Miller Fund, $\mathrm{O}=$ Ostrom Fund, $\mathrm{P}=$ Presidency Research Fund, $\mathrm{PF}=$ Prestage-Fenno Fund, $\mathrm{V}=$ Volcker, $\mathrm{W}=$ Women \& Politics Fund 


\section{Find your way to Washington with a little help.... www.apsanet.org}

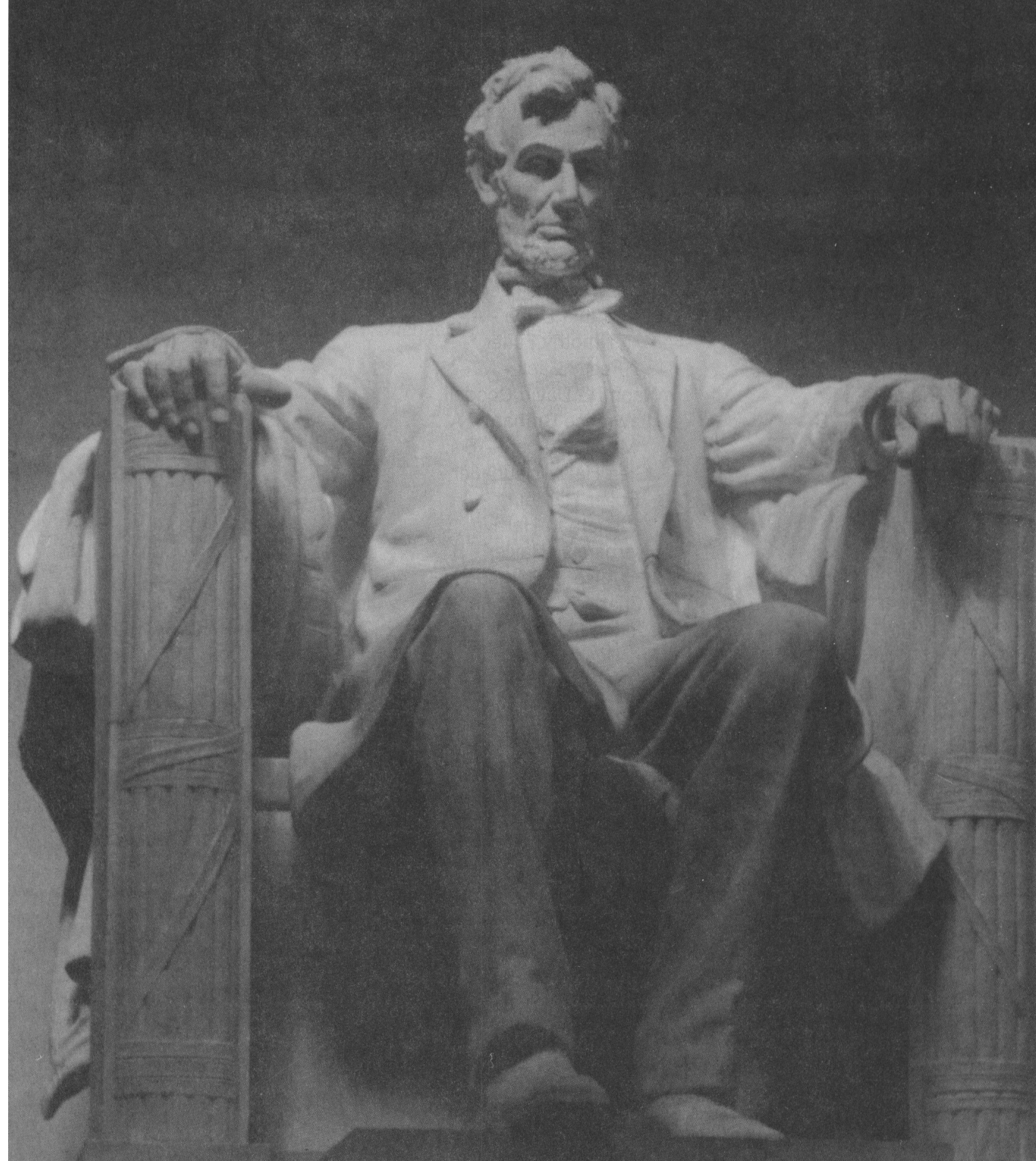

Washington 2000, August 31 - September 3 